\title{
Probing the dispersive and spatial properties of photonic crystal waveguides via highly efficient coupling from fiber tapers
}

\author{
P. E. Barclay, ${ }^{\text {a) }}$ K. Srinivasan, M. Borselli, and O. Painter \\ Department of Applied Physics, California Institute of Technology, Pasadena, California 91125
}

(Received 28 October 2003; accepted 10 May 2004)

\begin{abstract}
The demonstration of an optical fiber based probe for efficiently exciting the waveguide modes of high-index contrast planar photonic crystal (PC) slabs is presented. Fiber taper waveguides formed from standard silica single-mode optical fibers are used to evanescently couple light into the guided modes of a patterned silicon membrane. A coupling efficiency of $\sim 95 \%$ is obtained between the fiber taper and a PC waveguide mode suitably designed for integration with a previously studied ultrasmall mode volume high-Q PC resonant cavity [Srinivasan et al., Appl. Phys. Lett. 83, 1915 (2003)]. The micron-scale lateral extent and dispersion of the fiber taper is used as a near-field spatial and spectral probe to study the profile and dispersion of PC waveguide modes. (C) 2004 American Institute of Physics. [DOI: 10.1063/1.1767954]
\end{abstract}

While several initial applications of planar photonic crystals $(\mathrm{PCs})^{2}$ have been demonstrated, such as extremely small lasers ${ }^{1}$ and micro-optical add-drop filters, ${ }^{3}$ a significant barrier to further development of useful PC devices has been the difficulty in coupling light into and out of the PC. The small mode profiles, a result of the high refractive index of semiconductor materials from which high-index contrast PCs are typically created, and the complex optical phase within periodic structures make it difficult to use conventional coupling methods from optical fibers or free-space beams. ${ }^{4}$ In this letter, we demonstrate that by using the dispersive properties inherent to planar PCs, one may evanescently couple light in an efficient manner from silica optical fiber tapers, into a high-index contrast planar PC waveguide. ${ }^{7-9}$ Efficient contradirectional coupling is obtained to a photonic crystal waveguide (PCWG) mode designed for integration with a recently demonstrated high-Q PC cavity, ${ }^{1}$ and by utilizing the micron-scale lateral size and dispersion of the fiber taper this coupling technique is shown to be useful for mapping the band structure and spatial profile of PCWG modes.

The optical coupling scheme used in this work is shown schematically in Figs. 1(a) and 1(b). An optical fiber taper, formed by heating and stretching a standard single-mode silica fiber, is placed above and parallel to a PCWG. The fiber diameter changes continuously along the length of the fiber taper, reaching a minimum diameter on the order of the wavelength of light. Light that is initially launched into the core-guided fundamental mode of the optical fiber is adiabatically converted in the taper region of the fiber into the fundamental air-guided mode, allowing the evanescent tail of the optical field to interact with the PCWG; coupling occurs to phase-matched PCWG modes which share a similar momentum component down the waveguide at the frequency of interest. In order to probe the planar PC chip, the fiber taper is mounted in a " $u$ "-shaped configuration such that it forms an $\sim 10 \mathrm{~mm}$ straight segment at its midsection [Fig. 1(a)]. The mounted fiber taper is then placed on a vertical axis stage driven by a de motor with $50 \mathrm{~nm}$ encoder resolution, allowing the taper to be accurately positioned at varying

a) Author to whom correspondence should be addressed; electronic mail:
pbarclay@caltech.edu heights above individual PCWG devices [Figs. 1(b) and 1(c)]. The overall insertion loss in the mounted fiber tapers used here was less than $10 \%$, and more controlled formation of fiber tapers has shown that insertion loss of $<0.1 \mathrm{~dB}$ is possible. $^{10}$

The PCWGs used in this work were fabricated from a silicon-on-isulator (SOI) wafer. The air holes were patterned in the $340 \mathrm{~nm}$ thick silicon $(\mathrm{Si})$ layer using standard electron-beam lithography and dry etching techniques, after which the suspended membrane structure was created by selectively removing the $2 \mu \mathrm{m}$ thick underlying silicon dioxide layer using a hydorfluoric acid wet etch. An additional isolation etch, a few microns in depth, was performed to remove material surrounding the linear array of PCWGs [Fig. $1(c)]$. Along with the curvature of the fiber taper loop, this

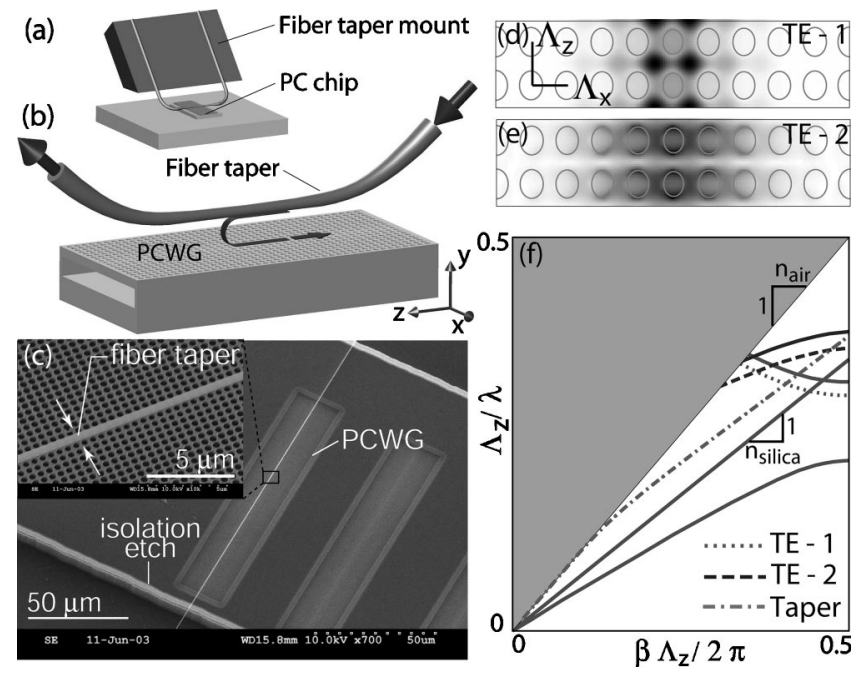

FIG. 1. (a) Schematic of the taper probe mount and (b) the coupling scheme. (c) Scanning electron microscope (SEM) images showing a fabricated PCWG with a fiber taper positioned at its center. Magnetic field profiles of the (d) TE-1 and (e) TE-2 PCWG modes. (f) Approximate (effective index calculation) two-dimensional band structure of a compressed square lattice $\left(\Lambda_{x} / \Lambda_{z} \sim 0.8\right)$ of air holes with radius $r=0.35 \Lambda_{x}$ for modes with TE-like polarization and dominant Fourier components in the $\Gamma-X(\hat{z})$ direction. Typical PCWG defect mode and fiber taper dispersion is also shown. 

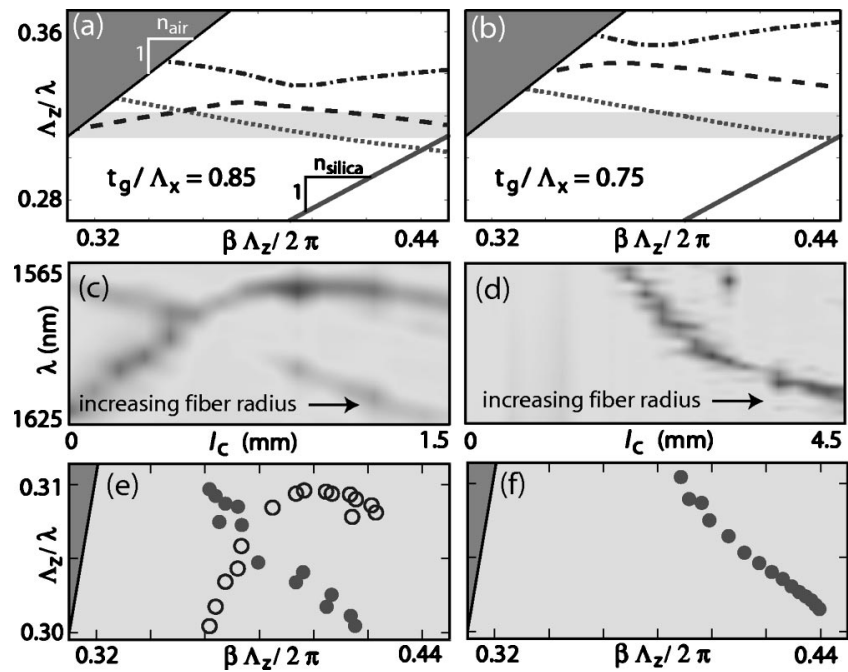

FIG. 2. 3D FDTD calculated dispersion of the TE-1 (dotted line), TE-2 (dashed line), and TM-1 (dot-dashed line) modes for the (a) unthinned $\left(t_{g}\right.$ $=340 \mathrm{~nm})$, and $(\mathrm{b})$ thinned $\left(t_{g}=300 \mathrm{~nm}\right)$ graded lattice PCWG membrane structure $\left(n_{\mathrm{Si}}=3.4\right)$. Measured transmission through the fiber taper as a function of wavelength and position along the tapered fiber for (c) unthinned sample and (d) thinned sample (different tapers were used for the thinned and unthinned samples, so the transmission vs $l_{c}$ data cannot be compared directly). Transmission minimum (phase-matched point) for each mode in the (e) unthinned and (f) thinned sample as a function of propagation constant. In (a) and (b), the lightly shaded regions correspond to the tuning range of the laser source used.

ensured that the fiber taper mode would only evanescently interact with the PCWG region.

Although the evanesent coupling scheme is not specific to a given defect geometry or lattice, the waveguide in this work was chosen to consist of a linear defect along the $\Gamma-X$ direction of a compressed square lattice $\left(\Lambda_{x}=0.4 \mu \mathrm{m}\right.$, $\Lambda_{z}=0.5 \mu \mathrm{m}$ ) formed by introducing a lateral grade in the hole radius [Figs. 1(c)-1(e)]. The choice of grading and lattice compression was motivated by previous work studying the design and integration of waveguides and ultrasmall mode volume, high-Q resonant cavities. This type of PC waveguide supports a mode (the TE-1 mode described below) which has a transverse overlap factor of greater than $98 \%$ with that of the high-Q resonant cavity studied in Ref. 1 , and initial finite-difference time-domain (FDTD) simulations of the coupled waveguide-cavity system indicate that efficient loading of the high-Q cavity can be obtained. ${ }^{8}$ Figure 1(f) shows an approximate band structure of TE-like (electric field predominantly in the plane of the slab) modes of the host compressed square lattice PC slab whose dominant Fourier components are in the direction of the waveguide, and which will consequently couple most strongly with the fundamental fiber taper mode. ${ }^{8}$ Superimposed upon this band structure are the important donor-type defect waveguide modes: (i) a defect mode with negative group velocity which comes off of the conduction band edge (TE-1), and (ii) a defect mode with positive group velocity which comes off of a second order (in the vertical direction) valence bandedge (TE-2). A typical fiber taper dispersion curve is also shown, lying between the air and silica light lines.

In Figs. 2(a) and 2(b), three-dimensional (3D) FDTD simulations were used to accurately calculate the PCWG band structure in the regions where phase matching to the fiber taper mode occurs. The FDTD-calculated in-plane magnetic field profiles of the TE-1 and TE-2 PCWG modes Downloaded 14 Dec 2005 to 131.215.225.171. Redistribution subject (taken in the midplane of the dielectric slab) near their respective phase-matching points are shown in Figs. 1(d) and 1(e). Although one can couple to either of the TE-1 or TE-2 modes, ${ }^{9}$ the TE- 1 mode is of primary interest here because of its fundamental nature in the vertical direction and its similar properties to that of the high-Q cavity mode of Ref. 1 .

In order to probe the band structure of the PCWG a scanning external cavity laser with a $1565-1625 \mathrm{~nm}$ wavelength range was connected to the fiber taper input. The transmitted power through the fiber taper section and the backreflected power into the fiber from the taper-PCWG coupling section were both monitored. By varying the position along the fiber taper of the interaction region between the PCWG and taper (as measured by $l_{\mathrm{c}}$, the distance from the fiber taper diameter minimum), the diameter $(d)$ of the fiber taper, and hence the propagation constant $(\beta)$ of the fiber taper mode interacting with the PCWG mode, could be tuned. Tuning from just below the air light-line $(d=0.6 \mu \mathrm{m}$, $\left.n_{\text {eff }}=\beta c / \omega \sim 1.05\right)$, to just above the silica light line ( $d$ $=4.0 \mu \mathrm{m}$ and $n_{\text {eff }} \sim 1.40$ ) was possible. Figure 2(c) shows the taper transmission as a function of wavelength and sample position $\left(l_{c}\right)$ when the taper is centered above the PCWG at a height $g \sim 700 \mathrm{~nm}$ from the PCWG surface. Resonances corresponding to both the TE-1 counter- and TE- 2 copropagating modes can be identified. SEM measurements of the taper diameter as a function of position $\left(l_{c}\right)$ were used to calculate the propagation constant of each resonance, allowing the PCWG modes' dispersion to be plotted [Fig. 2(e)]. The measured band structure is in close agreement with the FDTD calculated dispersion of Fig. 2(a), replicating both the negative group velocity of the TE-1 mode and the anticrossing behavior of the odd vertical parity TE-2 and TM-1 modes. Figures 2(d) and 2(f) show analogous data obtained by probing the sample after the Si slab thickness $t_{g}$ has been thinned to better isolate the TE-1 mode in $\omega-\beta$ space. As predicted by the FDTD simulation [Fig. 2(b)], the TE-1 mode is seen to shift slightly higher in frequency due to the sample thinning whereas the higher-order TE-2 mode shifts more quickly with slab thickness and is effectively "frozen" out of the laser scan range.

The dependence of the guided wave coupling on coupling strength and the overall efficiency of the coupling process were studied by varying the gap between the fiber taper and PCWG. Figure 3(a) shows the transmission through the fiber taper, with the coupling region occurring at a taper diameter of $d=1.9 \mu \mathrm{m}$, for varying taper heights above the thinned PCWG. Figure 3(b) shows the same measurement, but with the coupling region occurring at a taper diameter of $d=1.0 \mu \mathrm{m}$ and for a PCWG with slightly smaller nominal hole size. In both cases, the central resonance feature occurring around $\lambda=1600 \mathrm{~nm}$ is the coupling to the TE- 1 mode of the PCWG. In the $d=1.9 \mu \mathrm{m}$ case the minimum transmission $\left(T_{\min }\right)$ drops below $1 \%$ at the phase matching wavelength of $1602 \mathrm{~nm}$, and recovers to close to unity off resonance. The taper-PCWG gap $(g)$ dependence of $T_{\min }$ shown in Fig. 3(c) has the hyperbolic functional form $\tanh ^{2}\left(\kappa_{\perp} L_{c}\right)$ characteristic of contradirectional coupling ${ }^{11}$ for an effective coupling strength $\left(\kappa_{\perp} L_{c}\right)^{12}$ which depends exponentially on $g$ [Fig. 3(c) inset]. The degree of ideality of this coupling scheme $(\eta)$ can be estimated by subtracting the offresonance scattering loss (the scattering loss is expected to be broadband) from $1-T_{\min }$, and is plotted as a function of $g$ to AIP license or copyright, see http://apl.aip.org/apl/copyright.jsp 

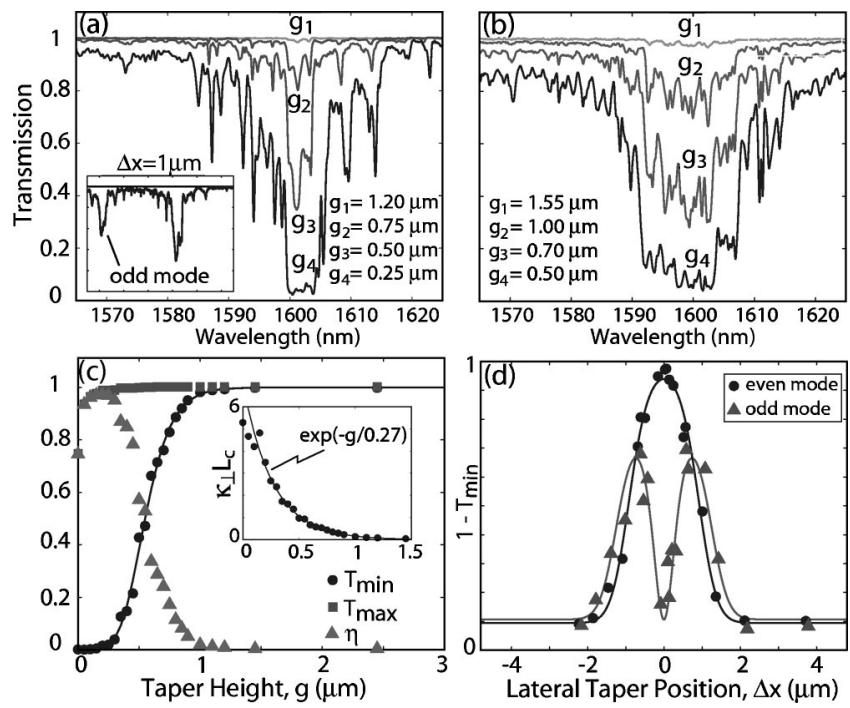

FIG. 3. Coupling characteristics from the fundamental fiber taper mode to the TE-1 PCWG mode of the thinned sample $\left(t_{g}=300 \mathrm{~nm}\right)$. Transmission vs wavelength for a (a) $1.9 \mu \mathrm{m}$ and (b) $1.0 \mu \mathrm{m}$ diameter taper coupling region for varying taper-PCWG gap, $g$. [(a) inset] Transmission vs wavelength for $\Delta x \sim 1 \mu \mathrm{m}$, same axes as (a). (c) $T_{\min }, T_{\max }$ (off-resonance), and $\eta$ vs $g$ with a $1.9 \mu \mathrm{m}$ diameter fiber taper coupling region. [(c) inset] Effective coupling strength $\left(\kappa_{\perp} L_{c}\right)$ vs $g$. (d) $1-T_{\min }$ vs lateral position $(\Delta x)$ of the $1.0 \mu \mathrm{m}$ diameter fiber taper relative to the center of the PCWG $(g=100 \mathrm{~nm})$. Transmission in (a)-(d) has been normalized to the transmission through the fiber taper in absence of the PCWG.

in Fig. 3(c) (triangles). The maximum $\eta$ occurs at a gap height of $g=250 \mathrm{~nm}$ and is $>95 \%$ in this case. This near unity coupling efficiency was further confirmed by studying the back-reflected power in the fiber taper, and is reported elsewhere. ${ }^{13}$

The coupler bandwidth was $20 \mathrm{~nm}$ for coupling with small diameter taper regions $(d \sim 1.0 \mu \mathrm{m})$ and less than $10 \mathrm{~nm}$ for coupling to regions of large taper diameter $(d$ $\sim 1.9 \mu \mathrm{m})$. This effect has two main contributions: the variation of the TE-1 PCWG mode group velocity at different points in the band structure $\left(\left|n_{g}\right|=|c \delta \beta / \delta \omega| \sim 4-6\right)$, and the variation in the taper diameter and hence propagation constant along the length of the $100 \mu \mathrm{m}$ PCWG $[\delta \beta / \delta d$ $\sim(0.084,0.36) \omega / c \mu \mathrm{m}^{-1}$ for $d=(1.9$ and 1.0$\left.) \mu \mathrm{m}\right]$.

The micron-scale lateral extent of the fiber taper may also be used as a near-field probe of the localized nature of the PCWG modes. Figure 3(d) shows the coupling dependence of the TE-1 PCWG mode as a function of lateral displacement of the taper from the center of the PCWG $(\Delta x)$.
The full width at half-maximum of $1-T_{\min }$ vs $\Delta x$ (for $1.0 \mu \mathrm{m}$ diameter taper coupling region) was measured to be $\sim 1.84 \mu \mathrm{m}$, in close agreement with the value $(2.08 \mu \mathrm{m})$ obtained using a simple coupled mode theory ${ }^{8}$ incorporating the fields of the FDTD calculated TE-1 mode and the analytically determined fiber mode. When the taper is displaced laterally, coupling to a second resonance [see Fig. 3(a) inset] is observed. This resonance has dispersion which is similar to the TE-1 mode's, but shifted $\sim 30 \mathrm{~nm}$ lower in wavelength, and corresponds to coupling to the odd (about $x$ ) counterpart to the TE-1 mode. The coupling is a result of the broken mirror symmetry about $x$ for $|\Delta x|>0$, and is a further demonstration of the local nature of the taper probe.

To summarize, evanescent coupling from an optical fiber taper to a highly confined donor-type photonic crystal waveguide mode has been demonstrated with $\sim 95 \%$ efficiency and over bandwidths as large as $1.3 \%$ of the center wavelength. This coupling technique is not only extremely efficient, but allows for the rapid optical probing of the spatial and dispersive properties of photonic crystal devices throughout the two-dimensional plane of a microchip. It is envisioned that this preliminary demonstration of a wafer scale optical probe for high-index contrast PCs will enable further experiments employing planar PCs.

One of the authors (K.S.) would like to thank the Hertz foundation and M.B. the Moore Foundation for financial support.

${ }^{1}$ K. Srinivasan, P. E. Barclay, O. Painter, J. Chen, A. X. Cho, and C. Gmachl, Appl. Phys. Lett. 83, 1915 (2003).

${ }^{2}$ E. Yablonovitch, Phys. Rev. Lett. 58, 2059 (1987).

${ }^{3}$ S. Noda, A. Chutinan, and M. Imada, Nature (London) 407, 608 (2000).

${ }^{4}$ M. Notomi, A. Shinya, K. Yamada, J. Takahashi, C. Takahashi, and I. Yokohama, IEEE J. Quantum Electron. 38, 736 (2002).

${ }^{5}$ C. H. Bulmer and M. G. F. Wilson, IEEE J. Quantum Electron. 14, 741 (1978).

${ }^{6}$ J. Knight, G. Cheung, F. Jacques, and T. Birks, Opt. Lett. 22, 1129 (1997).

${ }^{7}$ W. Kuang, C. Kim, A. Stapleton, and J. O'Brien, Opt. Lett. 27, 1604 (2002).

${ }^{8}$ P. E. Barclay, K. Srinivasan, and O. Painter, J. Opt. Soc. Am. B 20, 2274 (2003).

${ }^{9}$ P. E. Barclay, K. Srinivasan, M. Borselli, and O. Painter, IEEE Electron Device Lett. 39, 842 (2003).

${ }^{10}$ R. P. Kenny, T. A. Birks, and K. P. Oakley, IEEE Electron Device Lett. 27, 1654 (1991).

${ }^{11}$ P. Yeh and H. F. Taylor, Appl. Opt. 19, 2848 (1980).

${ }^{12}$ H. Kogelnik, Bell Syst. Tech. J. 55, 109 (1976).

${ }^{13}$ P. E. Barclay, K. Srinivasan, M. Borselli, and O. Painter, Opt. Lett. 29, 697 (2004). 